\title{
Extracorporeal Membrane Oxygenation as a Bridge to Initial Medical Therapy in a Patient With Decompensated Pulmonary Arterial Hypertension Presenting With Biventricular Failure
}

\author{
Donovan Mabe ${ }^{\mathrm{a}}$, Oksana A. Shlobin ${ }^{\mathrm{b}}$, Linda Bogar ${ }^{\mathrm{b}}$, Steven D. Nathan ${ }^{\mathrm{b}}$, \\ A. Whitney Brown ${ }^{\text {b }}$ Kareem Ahmad ${ }^{\text {b }}$, Shambhu Aryal ${ }^{\mathrm{b}}$, \\ Charles Murphy ${ }^{\mathrm{b}}$, Christopher S. King, c
}

\begin{abstract}
Extracorporeal membrane oxygenation (ECMO) can be used as a bridge to medical therapy in decompensated right heart failure due to pulmonary arterial hypertension (PAH). A middle-aged man with previously undiagnosed pulmonary hypertension was successfully bridged to intravenous prostanoid therapy with venoarterial ECMO support after presenting with cardiogenic shock and hypoxemic respiratory failure. Although the patient initially had biventricular failure, PAH was suspected due to underlying mixed connective tissue disease and disproportionate right ventricular dysfunction. On occasion, patients with PAH may present with biventricular failure. A high index of suspicion for PAH and serial reassessment of left ventricular function following correction of shock may demonstrate improvement in left ventricular function, allowing for initiation of pulmonary vasodilator therapy.
\end{abstract}

Keywords: ECMO; Venoarterial ECMO; Pulmonary arterial hypertension; Prostanoids

\section{Introduction}

Decompensated pulmonary arterial hypertension (PAH) is associated with a high morbidity and mortality. In-hospital mortality is estimated to be $30-40 \%$ for patients with PAH admitted to the intensive care unit (ICU) for decompensated right heart failure [1]. Successful management of decompensated $\mathrm{PAH}$ requires a high index of suspicion and prompt initiation

\footnotetext{
Manuscript submitted June 25, 2019, accepted August 26, 2019

${ }^{a}$ Walter Reed National Military Medical Center, Bethesda, MD, USA bInova Fairfax Hospital, Falls Church, VA, USA

${ }^{\mathrm{c} C}$ Corresponding Author: Christopher S. King, Inova Fairfax Hospital, Falls Church, VA, USA. Email: christopher.king@inova.org
}

of treatment. Extracorporeal membrane oxygenation (ECMO) has been successfully utilized as a bridge to lung transplant in end-stage PAH and as a bridge to recovery in PAH patients with a reversible insult, such as infection. Reports of ECMO as a bridge to initiation of pulmonary vasodilator therapy in a de novo presentation of PAH are limited $[2,3]$. We present a patient with previously undiagnosed $\mathrm{PAH}$ who presented to our institution with profound hypoxemic respiratory failure and cardiogenic shock, who was successfully bridged to intravenous (IV) prostanoid therapy using venoarterial (VA) ECMO.

\section{Case Report}

A 54-year-old man with a history of mixed connective tissue disease presented to the emergency department with acute hypoxemic respiratory failure and shock. His chest radiograph revealed a right upper lobe infiltrate and small bilateral pleural effusions (Fig. 1). His initial partial pressure

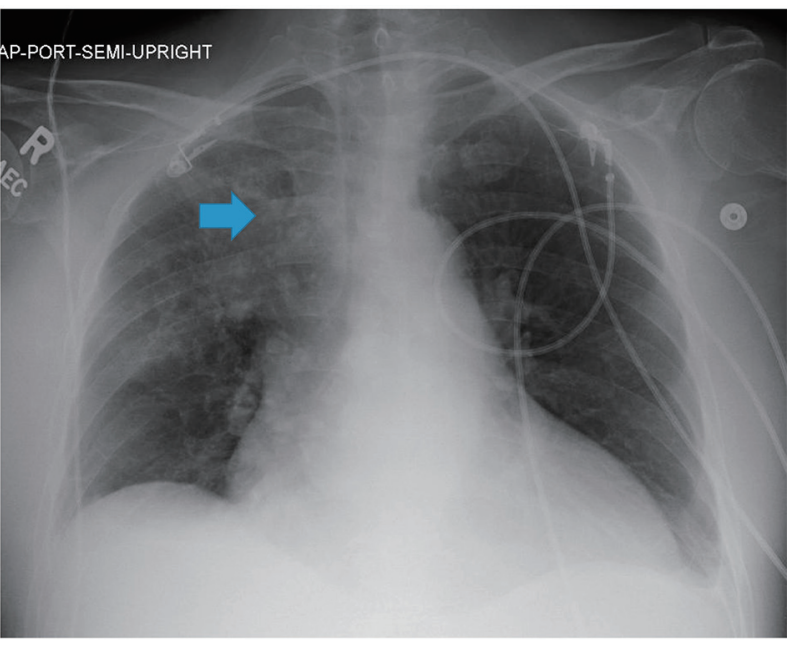

Figure 1. Portable anterior-posterior chest radiograph from admission revealed a right upper lobe infiltrate (blue arrow) and small bilateral pleural effusions. 

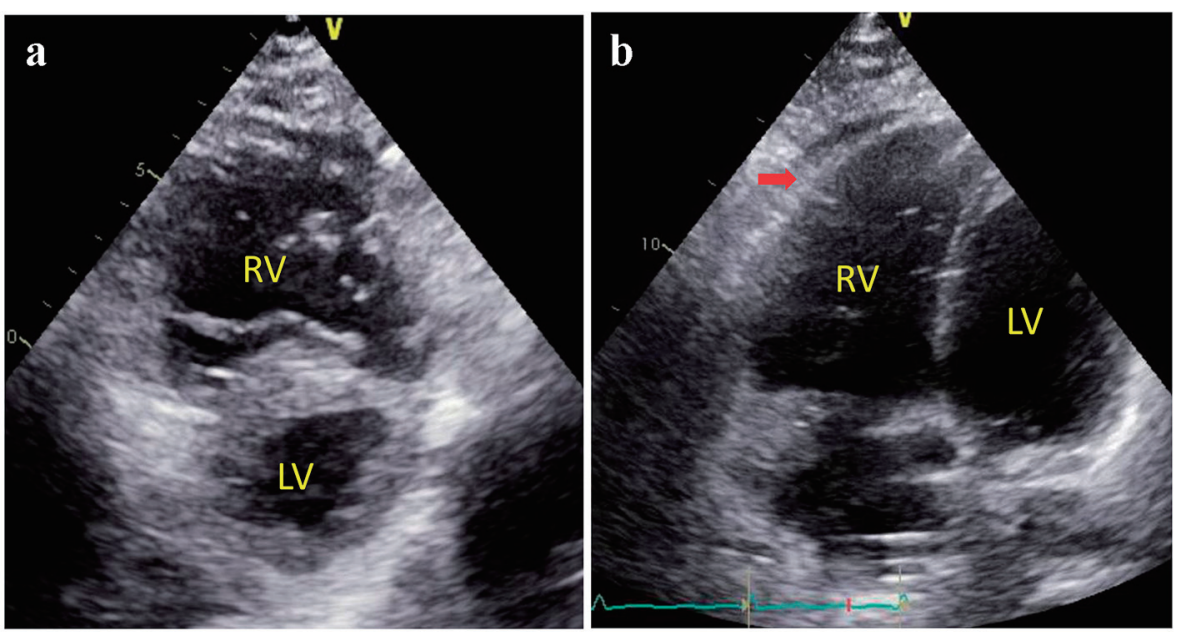

Figure 2. Parasternal short axis view (a) and apical four chamber view (b) of transthoracic echocardiogram revealed a markedly enlarged right ventricle and a flattened "D-shaped" septum consistent with right ventricular volume and pressure overload.

of arterial oxygen $\left(\mathrm{PaO}_{2}\right)$ on room air was $37 \mathrm{~mm} \mathrm{Hg}$. His labs were notable for acute kidney injury with a creatinine of 1.6, elevated liver function tests suggestive of shock liver, and lactic acidosis (4.3). The patient was intubated for severe hypoxemia which was complicated by a brief cardiac arrest. Bedside transthoracic echocardiography (TTE) revealed marked right ventricular dilation and evidence of a patent foramen ovale (PFO) on a bubble study (Fig. 2a and b). Computed tomography (CT) pulmonary angiography was negative for pulmonary embolism, but confirmed a right upper lobe infiltrate consistent with pneumonia and bilateral pleural effusions (Fig. 3). The patient remained profoundly hypoxemic and developed hypotension requiring vasopressor use. The decision was made to place the patient on VA ECMO for treatment of cardiopulmonary failure.

Peripheral VA ECMO was urgently initiated using the CardioHelp device with a drainage catheter in the right femoral vein and the reinfusion catheter positioned in the right femoral artery. Subsequent arterial blood gas with ECMO flow of $3.7 \mathrm{~L} / \mathrm{min}$, sweep of $1.0 \mathrm{~L} / \mathrm{min}$ and fraction of in-

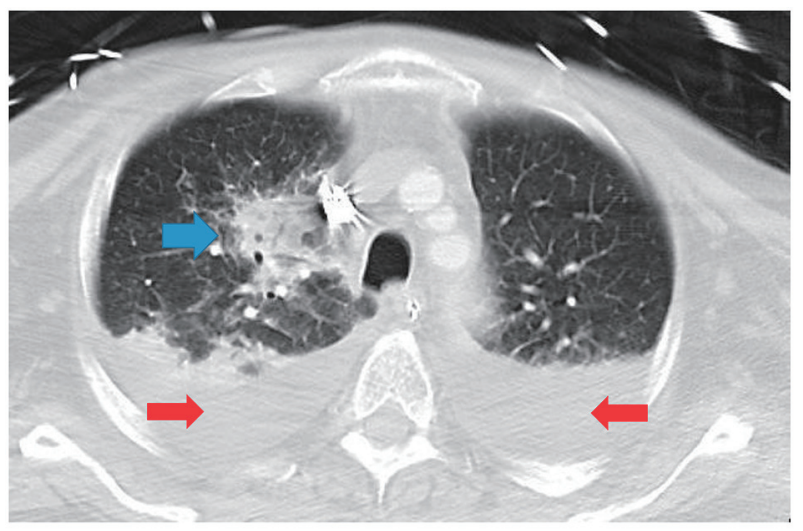

Figure 3. CT angiogram showing a necrotizing right upper lobe pneumonia (blue arrow) and small bilateral pleural effusions (red arrows). CT: computed tomography. spired oxygen $\left(\mathrm{FiO}_{2}\right)$ of 0.55 showed a $\mathrm{PaO}_{2}$ of 105 . He was started on empiric antibiotic therapy for community-acquired pneumonia as well as stress dose steroids. Transesophageal echocardiography at the time of ECMO cannulation revealed biventricular failure with a left ventricular ejection fraction (LVEF) of about $15 \%$. Inhaled nitric oxide (iNO) as well as IV epinephrine and milrinone were initiated. Serial echocardiography indicated improving LVEF but persistent right ventricular dysfunction over the next few days. Given the high index for suspicion of pulmonary arterial hypertension due to the disproportionately enlarged right ventricle (RV) in the context of previously diagnosed mixed connective tissue disease, IV epoprostenol was initiated on ECMO day 4 and titrated to 15 $\mathrm{ng} / \mathrm{kg} / \mathrm{min}$. The iNO and hemodynamic support were gradually weaned and he was decannulated from ECMO support after 9 days.

Due to profound weakness from critical illness polyneuropathy, he required tracheostomy and gastrostomy tube placement. As he improved during the hospitalization of approximately 2 months, these were removed. He was converted from IV epoprostenol to IV treprostinil due to lifestyle considerations. Prior to discharge, his LVEF improved to $60-65 \%$ and right heart catheterization on treprostinil therapy at $40 \mathrm{ng} / \mathrm{kg} /$ min demonstrated moderately severe but compensated PAH with a right atrial pressure of $5 \mathrm{~mm} \mathrm{Hg}$, a mean pulmonary artery pressure (mPAP) of $43 \mathrm{~mm} \mathrm{Hg}$, a pulmonary capillary wedge pressure (PCWP) of $9 \mathrm{~mm} \mathrm{Hg}$ and a cardiac index of $3.1 \mathrm{~L} / \mathrm{min} / \mathrm{m}^{2}$. At 6-month follow-up, he was reporting World Health Organization functional class 2 symptoms and living independently without the need for supplemental oxygen. This report is retrospective and therefore did not necessitate institutional review board approval.

\section{Discussion}

Extracorporeal support is being increasingly utilized in the management of pulmonary hypertension. The role of ECMO 
support as a bridge to transplant in advanced PAH is well documented in the medical literature. ECMO, both venovenous and venoarterial configurations, has also been utilized as a bridge to recovery from a reversible insult in the context of established PAH already on therapy. Few reports have described the use of ECMO as a bridge to vasodilator therapy in previously untreated PAH presenting with cardiogenic shock $[2,3]$.

Multiple factors are required to successfully implement ECMO support in the context of newly diagnosed PAH. Early recognition and correct diagnosis of the disease are crucial. To establish the diagnosis of PAH requires a high index of suspicion and consideration of clinical context. Our patient could easily have been labeled as having pneumonia and septic shock alone, but without initiation of pulmonary vasodilator therapy, he would have been unlikely to be successfully liberated from ECMO support. Clinicians should have a heightened suspicion in patients with risk factors for PAH including a history of connective-tissue disease or stigmata thereof, human immunodeficiency syndrome, or a history of stimulant use, although these are not mandatory for the development of the disease. Physical exam signs of long-standing right heart failure including lower extremity edema, jugular venous distention, and ascites may be present. Bedside TTE can be used to screen for evidence of pulmonary hypertension. Dilation of the right ventricle and septal flattening often are present in patients with significant PAH, and can be easily detected by intensivists on bedside echocardiographic exams.

The presence of left ventricular (LV) dysfunction, as seen in our case, can obscure the correct diagnosis and further complicate the decision to initiate PAH therapy. Use of pulmonary vasodilator therapy is typically contraindicated in the setting of marked LV dysfunction as it may lead to pulmonary edema. The LV dysfunction in this case was thought to be multifactorial with myocardial stunning due to severe hypoxemia, acidosis and shock being the most likely causes. An additional consideration when contemplating VA ECMO is that an already depressed LV might decompensate further with the added afterload resulting from the retrograde outflow of the ECMO circuit into the aorta. The exact mechanisms for LV dysfunction in PAH are incompletely understood, but are commonly appreciated immediately after lung transplantation for PAH [4]. Decreased delivery of LV preload due to RV dysfunction over time may lead to some degree of LV atrophy. Additionally, the detrimental effects of RV pressure overload on the movement of the septum may contribute. In order to assess LV function accurately, it is important to limit the effects of afterload mismatch from the ECMO flow. This can be accomplished by real-time echocardiographic assessment while ECMO support is weaned. We performed serial "turn-down" trials demonstrating improving LV function prior to the initiation of pulmonary vasodilator therapy. Therapeutic options for patients with biventricular failure and pulmonary hypertension are extremely limited. Had we failed to recognize the reversible nature of the LV dysfunction in this case and not initiated PAH specific pulmonary vasodilator therapy, it is likely the patient's course would not have been reversed.

The patient we presented had several factors which may have contributed to his successful outcome. First, he had a reversible insult in the form of a pneumonia. While this infec- tion certainly contributed to his acute decompensation, it also caused him to seek medical care at a stage when his RV failure was less advanced. Additionally, the patient had a cardiac shunt due to a PFO, which led to refractory hypoxemic respiratory failure. While the PFO was responsible for the severity of his hypoxemia on presentation due to right-to-left shunting in the setting of acute RV decompensation, it may have been protective in the context of his PAH, providing a "pop-off" to unload his RV. Finally, our patient was treatment-naive and therefore "primed" for response to pulmonary vasodilator therapy. Appropriate patient selection prior to initiation of ECMO support in bridge-to-recovery patients with PAH is essential. Optimal candidates should have a readily reversible etiology of decompensation and/or suboptimally treated or treatment-naive PAH, and not treatment refractory disease.

\section{Conclusion}

The role of extracorporeal support in the treatment of PAH continues to expand. ECMO can be successfully utilized as a bridge to initiation of pulmonary vasodilator therapy in de novo PAH patients presenting with RV failure. It is important to be aware that in rare cases patients with PAH may demonstrate biventricular failure on presentation. In such situations, serial reassessment of LV function is critical, as improvement in LV function opens the door for pulmonary vasodilator therapy as a therapeutic strategy.

\section{Acknowledgments}

None to declare.

\section{Financial Disclosure}

No funding was received. None of the authors have disclosures relevant to this manuscript.

\section{Conflict of Interest}

None to declare.

\section{Informed Consent}

The manuscript has been sufficiently de-identified to protect the patient. He has since died and is unable to provide informed consent.

\section{Author Contributions}

All authors contributed to the editing of the manuscript. CK and DM wrote the manuscript and made the accompanying figures. 


\section{References}

3. Tsai MT, Hsu CH, Luo CY, Hu YN, Roan JN. Bridgeto-recovery strategy using extracorporeal membrane oxygenation for critical pulmonary hypertension complicated with cardiogenic shock. Interact Cardiovasc Thorac Surg. 2015;21(1):55-61.

4. Gupta S, Torres F, Bollineni S, Mohanka M, Kaza V. Left ventricular dysfunction after lung transplantation for pulmonary arterial hypertension. Transplant Proc. 2015;47(9):2732-2736. 